\title{
Microestructura y comportamiento plástico de perovsquitas conductoras protónicas de alta temperatura
}

\author{
M.J. LÓPEZ-ROBLEDO, J. RAMÍREZ-RICO*, J. MARTíNEZ-FERNÁNDEZ, A.R. DE ARELLANO-LÓPEZ, A. SAYIR* \\ Departamento de Física de la Materia Condensada, Universidad de Sevilla, 41012 Sevilla, ESPAÑA. \\ ${ }^{*}$ Case-Wertern Reserve University, Cleveland, OH, USA
}

\begin{abstract}
Se ha estudiado el comportamiento en fluencia de perovsquitas conductoras protónicas de alta temperatura fabricadas por fusión láser, en particular los sistemas $\mathrm{SrCe}_{0.9} \mathrm{Y}_{01} \mathrm{O}_{3-\delta}$ y $\mathrm{Sr}_{3} \mathrm{Ca}_{118} \mathrm{Nb}_{182} \mathrm{O}_{9-\delta}$. Se ha estudiado la microestructura antes y después de los ensayos mecánicos mediante Microscopía Electrónica de Barrido (MEB) y Difracción de Electrones Retrodispersados (Electron BackScattering Diffraction, EBSD), con el objetivo de correlacionar el comportamiento plástico de estos materiales con su evolución microestructural. Los materiales analizados tienen una estructura celular de granos alargados, separados por una fase amorfa, y presentan una fuerte textura cristalográfica bi-axial. Se han realizado ensayos de deformación a carga constante (fluencia) a diversas tensiones y temperaturas, en atmósfera inerte de Ar y en aire. Tras los ensayos, la fase intercelular fluye hacia el exterior de la muestra y se ha comprobado mediante EBSD que los granos rotan de forma rígida. El comportamiento en fluencia de estos materiales es independiente de la atmósfera de trabajo y se corresponde con un mecanismo de flujo viscoso de las células en la matriz amorfa, controlada por difusión en volumen.
\end{abstract}

Palabras clave: Conductor protónico, fluencia, EBSD, solidificación direccional

Microstructure and creep behaviour of high temperature proton conducting perovskites.

The creep behaviour of high temperature proton conducting perovskites $\mathrm{SrCe}_{0.9} \mathrm{Y}_{01} \mathrm{O}_{3-\delta}$ and $\mathrm{Sr}_{3} \mathrm{Ca}_{118} \mathrm{Nb}_{182} \mathrm{O}_{9-\delta}$ fabricated by laser fusion has been studied. Their microstructure has been studied both in the as-received and crept samples by means of Scanning Electron Microscopy (SEM) and Electron BackScattering Diffraction (EBSD), to correlate plastic behaviour with the evolution of the microstructure. The materials show a cellular structure consisting of elongated grains embedded in an amorphous phase and a strong bi-axial crystallographic texture. Deformation tests at constant stress (creep tests) have been performed both in Ar atmosphere and in air. Rigid grain rotation has been observed in the crept samples by EBSD. The amorphous phase flows outside the sample during creep. Plastic behaviour of these materials is independent of the environmental atmosphere and is consistent with a mechanism of viscous flow of the amorphous phase controlled by diffusion.

Keywords: Proton conductor, creep, EBSD, directional solidification.

\section{INTRODUCCIÓN}

Los conductores protónicos de alta temperatura (CPAT) tienen un gran interés en una amplia gama de aplicaciones electroquímicas, como por ejemplo en células de combustible, electrolitos de vapor, membranas para la separación de gas, células de hidrólisis, sensores de humedad y baterías de alta densidad. Desde que Iwahara y col. (1) descubrieran las propiedades de conducción protónica a alta temperatura de algunas perovsquitas basadas en el $\mathrm{SrCeO}_{3^{\prime}}$ el diseño y estudio de nuevos conductores protónicos ha acaparado mucho interés (2). Debido a que las condiciones de servicio de estos materiales son muy agresivas térmica y mecánicamente, es necesario un estudio profundo de sus propiedades mecánicas a alta temperatura. Las propiedades de resistencia en compresión han sido estudiadas por López-Robledo y col. (3), pero es conveniente conocer también las propiedades de fluencia de estos materiales (4), que determinarán su vida útil a largo plazo. Además, los estudios de fluencia pueden aportar información sobre los procesos de difusión que ocurren en estos materiales, lo que resulta de importancia en la sinterización. Este trabajo pretende arrojar algo de luz en este asunto, pues los estudios existentes en la literatura han sido realizados en policristales, donde la fluencia está controlada por difusión en las fronteras de grano (5). La particular microestructura de los materiales crecidos por solidificación direccional nos podría permitir estudiar la difusión en volumen, aspecto sobre el que no existen datos en la literatura.

\section{TÉCNICAS EXPERIMENTALES}

\subsection{Fabricación}

Nuestros CPAT se han fabricado por solidificación direccional, mediante la técnica de fusión zonal asistida por láser $(6,7)$. Esta técnica, descrita anteriormente (3), permite obtener materiales solidificados direccionalmente a partir de la fusión de cerámicos policristalinos con un láser de $\mathrm{CO}_{2}$. Éstos, por lo general, presentan unas excelentes propiedades mecánicas debido a la fuerte anisotropía de crecimiento (7). 
Las muestras de $\mathrm{SrCe}_{0.9} \mathrm{Y}_{0.1} \mathrm{O}_{3-\delta}$ se fabricaron a partir de polvos policristalinos de $\mathrm{SrCO}_{3}$ de alta pureza (99.999\% puro), $\mathrm{CeO}_{2}$ de alta pureza (99.999\% \%uro) y $\mathrm{Y}_{2} \mathrm{O}_{3}$ (99.99\% \%uro) suministrados por CERAC (tamaño de grano 325). El polvo fue mezclado y molido durante 40 horas. Después fue calcinado a $80{ }^{\circ} \mathrm{C}$ en aire durante 6 horas. Se deshicieron los aglomerados y se tamizó; el polvo resultante se compactó en frío obteniendo cilindros que fueron sinterizados en aire a $1500{ }^{\circ} \mathrm{C}$ durante 10 horas. Estas barras fueron utilizadas como precursores en el equipo de fusión zonal con láser. El haz láser se divide en dos, a $180^{\circ}$ uno del otro, y cada rayo se focaliza con lentes de ZnSe sobre la parte superior de la barra policristalina hasta formar una gota, que moja una semilla de $\mathrm{Al}_{2} \mathrm{O}_{3}$ de alta densidad (>99.9\%). La zona fundida así formada se mantiene por tensión superficial entre los dos sólidos. Luego se hace mover la barra policristalina a través de la zona de incidencia del láser, y el material funde y cristaliza unidireccionalmente. La velocidad de crecimiento fue de $500 \mathrm{~mm} / \mathrm{h}$ para todas las muestras, que tienen forma cilíndrica de aproximadamente $4 \mathrm{~mm}$ de diámetro y $100 \mathrm{~mm}$ de longitud. El proceso de fabricación para las muestras de $\mathrm{Sr}_{3} \mathrm{Ca}_{1.18} \mathrm{Nb}_{1.82} \mathrm{O}_{9-\delta}$ fue similar.

\subsection{Técnicas de caracterización}

Las muestras se cortaron en cilindros de $4 \mathrm{~mm}$ de diámetro y $5 \mathrm{~mm}$ de longitud y se sometieron a ensayos de compresión uniaxal a tensión constante (fluencia) mediante una máquina de ensayos prototipo (8). La temperatura de trabajo se escogió en torno a los $1300^{\circ} \mathrm{C}$, y el rango de cargas entre 40 y $200 \mathrm{MPa}$. Los ensayos se realizaron en atmósferas de Aire $\left(\mathrm{p}\left(\mathrm{O}_{2}\right)=0.27\right.$ atm) y de Ar, cambiando la atmósfera de trabajo durante el transcurso de la experiencia.

Tanto las muestras obtenidas tras el crecimiento como las sometidas a ensayos de compresión fueron cortadas con una sierra adiamantada y pulidas con pasta de diamante de hasta $1 \mu \mathrm{m}$ y con solución OPS (KOH + sílica coloidal), para su estudio en MEB y EBSD. Los estudios de MEB y EBSD se realizaron en un microscopio Philips XL-30 equipado con detectores de electrones secundarios, retrodispersados, microanálisis EDS y una cámara de difracción EBSD DigiView II. La técnica EBSD permite obtener diagramas de difracción electrónica por reflexión en un microscopio electrónico de barrido, de una pequeña área de $0.5 \times 0.5 \mu \mathrm{m}^{2}$, que es adquirido e indexado mediante software especializado. El haz de electrones puede barrerse sobre la muestra para obtener diagramas de difracción de puntos equiespaciados sobre su superficie, y obtener un mapa de orientaciones con resolución espacial. En nuestros estudios se adquirieron e indexaron más de 100,000 diagramas de difracción en un área de 90×250 $\mu \mathrm{m}^{2}$, tanto de las muestras recibidas como de las muestras sometidas a ensayos de fluencia. Aunque ambos materiales presentan estructura ortorrómbica con grupo espacial Pmna, la diferencia de los parámetros reticulares en la subcelda pseudocúbica está por debajo de la resolución del sistema EBSD usado en este estudio. Por este motivo se asumió una estructura de grupo espacial $P m \overline{3} m$, correspondiente al prototipo cúbico. Las equivalencias aproximadas para las direcciones reticulares en ambas estructuras son:

$$
\begin{aligned}
{[101]_{\mathrm{c}} } & \sim[100]_{\mathrm{o}} \\
{[010]_{\mathrm{c}} } & \sim[010]_{\mathrm{o}} \\
{[-101]_{\mathrm{c}} } & \sim[001]_{\mathrm{o}}
\end{aligned}
$$

Donde el subíndice c denota la red cúbica y el subíndice o se refiere a la estructura ortorrómbica. Los parámetros reticulares cumplen las relaciones aproximadas:

$$
\begin{aligned}
& a_{o} \sim c_{o} \sim \sqrt{2} a_{c} \\
& b_{o} \sim 2 a_{c}
\end{aligned}
$$

Pueden obtenerse más detalles sobre la técnica EBSD en la literatura (9).

\section{RESULTADOS Y DISCUSIÓN}

\subsection{Caracterización de las muestras crecidas por fusión zonal láser}

Las muestras presentan en los dos sistemas una estructura celular formada por granos alargados en una matriz amorfa (3). En la figura 1 se muestran diferentes micrografías de MEB correspondientes a la sección transversal de $\mathrm{SrCe}_{0.9} \mathrm{Y}_{0.1} \mathrm{O}_{3-8}$. La microestructura de las muestras de $\mathrm{Sr}_{3} \mathrm{Ca}_{1.18} \mathrm{Nb}_{1.82} \mathrm{O}_{9-\delta}$ es similar.

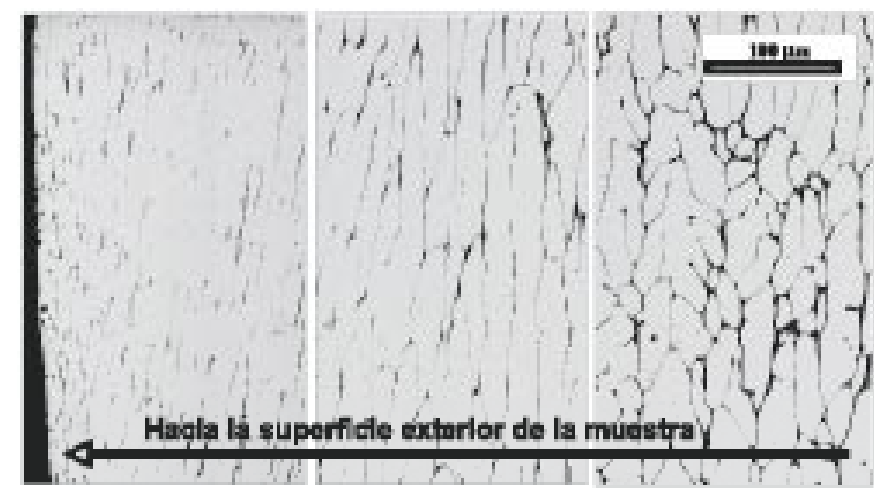

Fig. 1.- Micrografía electrónica de barrido de una sección transversal de $\mathrm{SrCe}_{0.9} \mathrm{Y}_{0.1} \mathrm{O}_{3-\delta^{\prime}}$ mostrando una estructura celular. La fase más oscura representa la fase amorfa. Se observa que la fracción en volumen de fase amorfa aumenta en la dirección radial.

En todas las muestras se observa que la fracción de volumen de fase amorfa disminuye en la dirección radial de la muestra, a medida que nos acercamos a la superficie exterior. Debido a que la radiación láser es absorbida en la superficie de la muestra, el interior se calienta por difusión térmica, de manera que se establece un gradiente de temperaturas en la dirección radial (10). Así, la temperatura en la superficie es mayor que en el centro de la muestra, lo que favorece la cristalización. La figura 2 muestra dos espectros EDS adquiridos en el interior de las células y en la región intercelular amorfa, observándose que en el segundo caso la concentración de Ce es mucho menor.

Los resultados de EBSD en las muestras sin deformar muestran una fuerte textura cristalina bi-axial, del tipo $\{001\}<110>$ referida al prototipo cúbico, como se representa en el diagrama de polos de la figura 3 . Esto significa que en la estructura ortorrómbica real coexisten seis variantes orientacionales o dominios cristalográficos, que son equivalentes en la estructura cúbica prototipo 


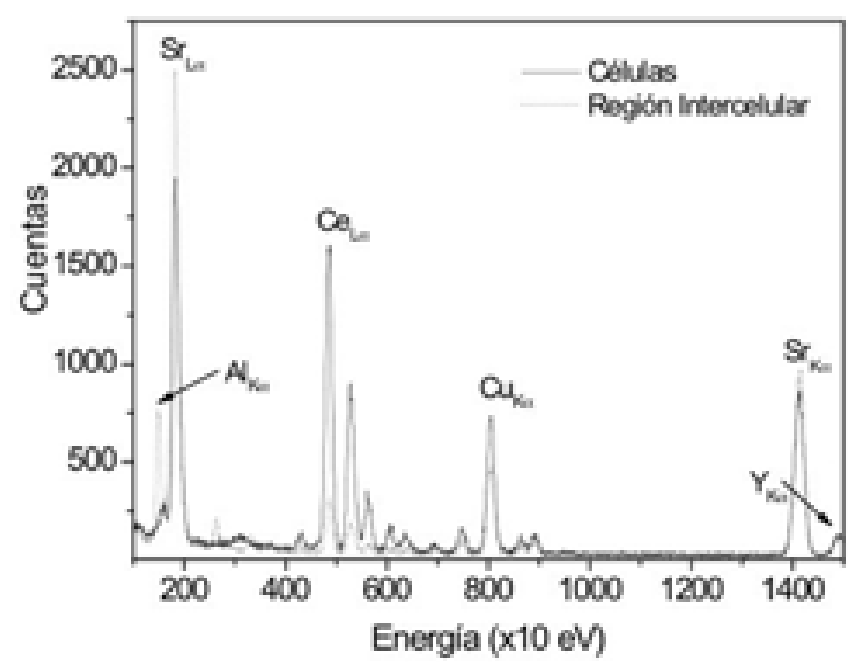

Fig. 2.- Espectros EDS de $\mathrm{SrCe}_{09} \mathrm{Y}_{01} \mathrm{O}_{3-\delta}$ adquiridos en el interior de las células y en la región intercelular amorfa. Se observa que la fase vítrea tiene un contenido menor de Ce.

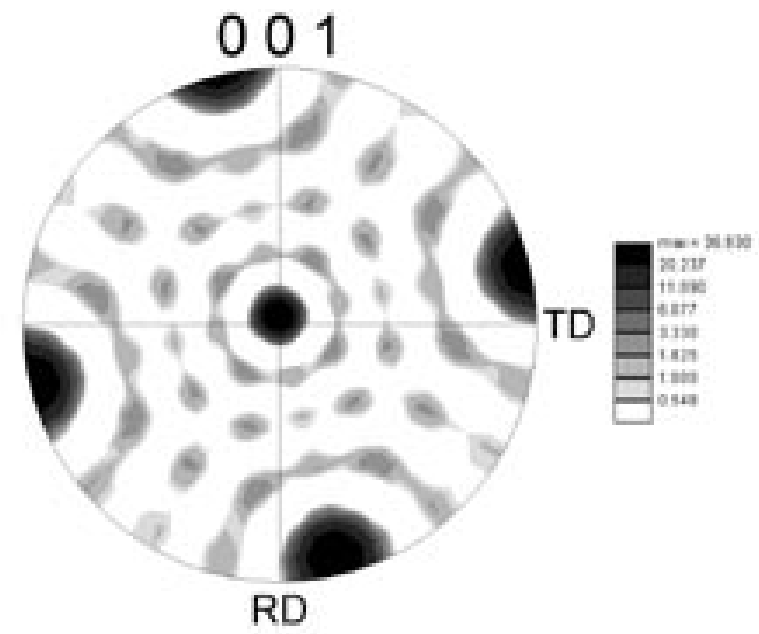

Fig. 3.- Diagramas de polos $\{001\}$ de una muestra de $\mathrm{SrCe}_{0.9} \mathrm{Y}_{0.1} \mathrm{O}_{3-\delta}$. Se observa una fuerte textura bi-axial $\{001\}<110>$ (escala logarítmica, unidades arbitrarias).

Las células tienen por tanto estructura de monocristal, todas con aproximadamente la misma orientación. Esta es una propiedad conocida de los materiales fabricados por solidificación direccional (10).

\subsection{Fluencia a alta temperatura}

Los ensayos de fluencia muestran que la velocidad de deformación estacionaria en estos materiales no depende de la presión parcial de oxígeno, lo que está de acuerdo con los resultados obtenidos por otros autores en compuestos similares (5). Se realizaron cambios de atmósfera durante el transcurso del ensayo, comprobándose que las condiciones de fluencia de nuestros materiales no cambian apreciablemente (figura 4).

Para obtener los exponentes de tensión n y la energía de activación $Q$ de estos materiales se realizaron saltos de carga y de temperatura durante el transcurso de las experiencias. Las curvas típicas de fluencia se observan en las figuras 5 y 6 .

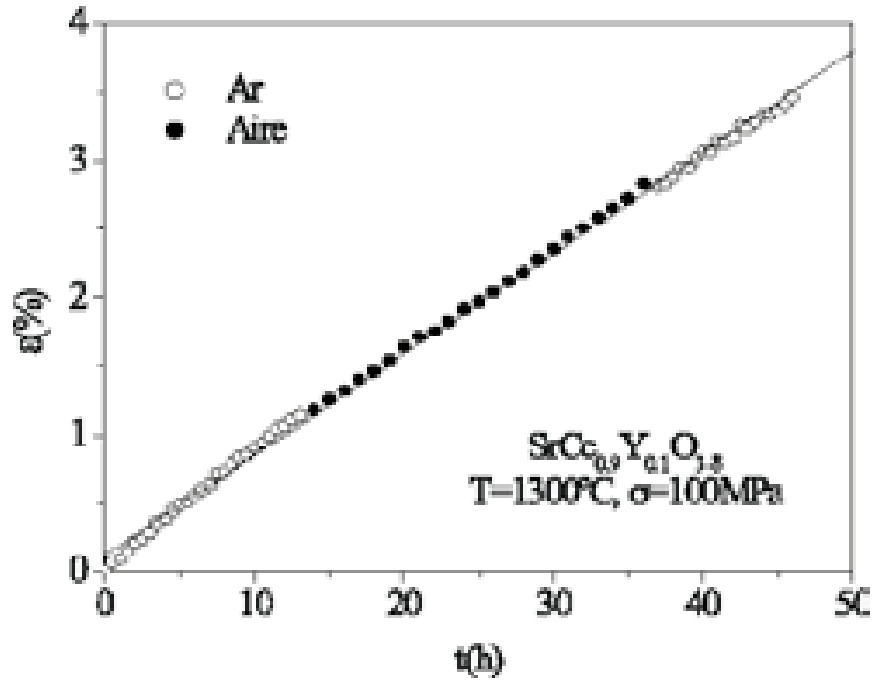

Fig. 4.- Curva de fluencia $\varepsilon(\mathrm{t})$ de una muestra de $\mathrm{SrCe}_{0.9} \mathrm{Y}_{0.1} \mathrm{O}_{3-\delta}$ con cambios de atmósfera durante el transcurso de la experiencia. La temperatura del ensayo es de $1300^{\circ} \mathrm{C}$, con una carga constante de 100MPa.

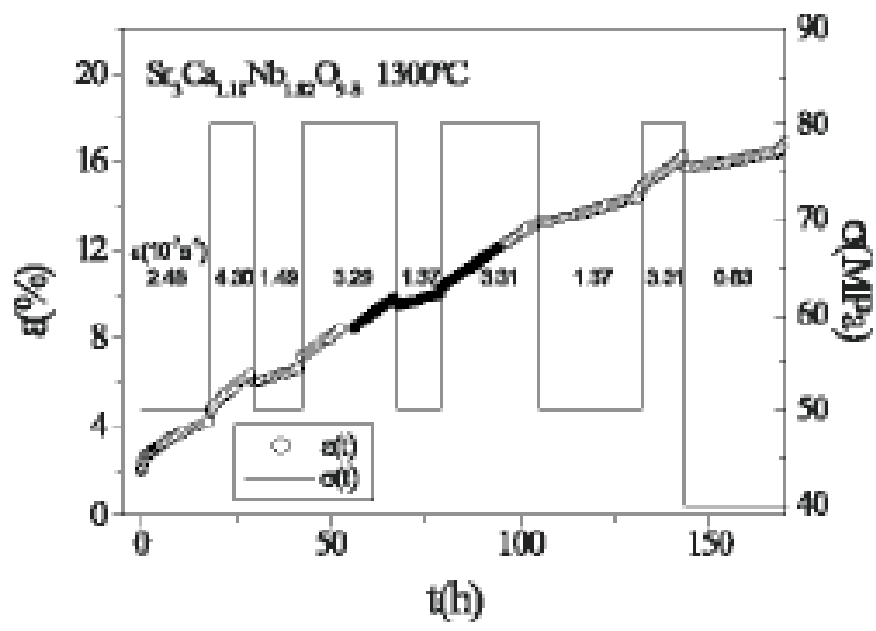

Fig. 5.- Curva de fluencia $\varepsilon(t)$ de una muestra de $\operatorname{SrCe}_{0.9} \mathrm{Y}_{0.1} \mathrm{O}_{3-\delta}$ con saltos de carga y cambios de atmósfera durante el transcurso de la experiencia. La temperatura del ensayo es de $1300^{\circ} \mathrm{C}$. Los puntos vacíos (o) corresponden a una atmósfera de $\operatorname{Ar}$ y los rellenos $(\bullet)$ a atmósfera de Aire.

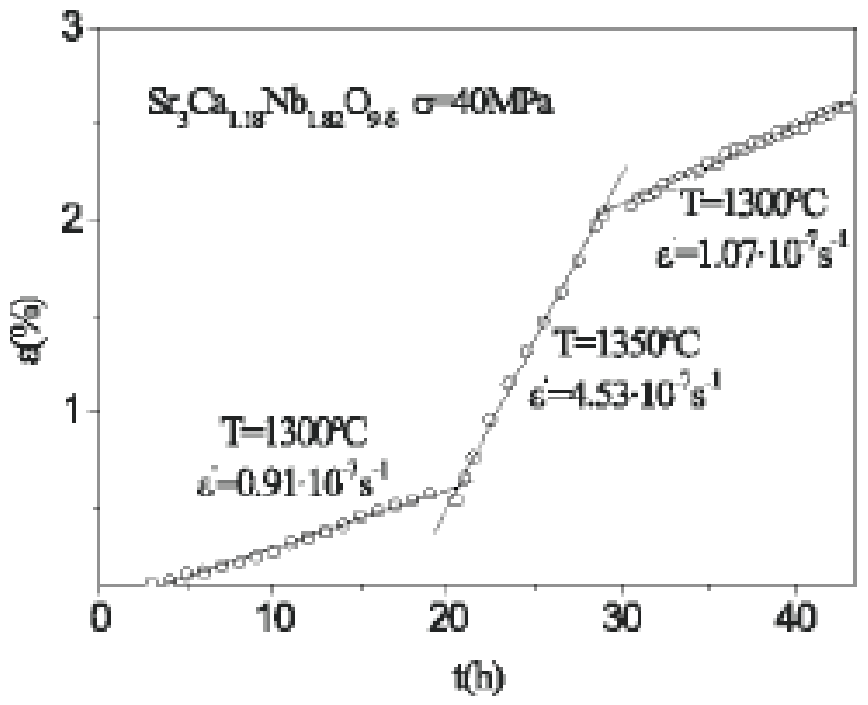

Fig. 6.- Curva de fluencia $\varepsilon(\mathrm{t})$ de una muestra de $\mathrm{SrCe}_{0.9} \mathrm{Y}_{0.1} \mathrm{O}_{3-\delta}$ con saltos de temperatura durante el transcurso de la experiencia. La carga es de $40 \mathrm{MPa}$. 
Se han determinado los exponentes de tensión $\mathrm{n}$ para todas las muestras estudiadas, y para el $\mathrm{Sr}_{3} \mathrm{Ca}_{118} \mathrm{Nb}_{182} \mathrm{O}_{9-}$ ${ }_{\delta}$ se ha determinado la energía de activación $Q$, con los resultados obtenidos de las curvas de fluencia. La figura 7 muestra el cálculo de la energía de activación, para la que encontramos un valor de $\mathrm{Q}=670 \mathrm{KJ} \cdot \mathrm{mol}^{-1}$. Este valor es superior al de $343 \mathrm{KJ} \cdot \mathrm{mol}^{-1}$ obtenido por Park y col. (5) para policristales de $\mathrm{BaCe}_{1-x} \mathrm{Y}_{\mathrm{x}} \mathrm{O}_{3-\mathrm{y}^{\prime}}$ que corresponde a un mecanismo de deformación controlado por deslizamiento de junta de grano acomodado por difusión, que probablemente ocurre en volumen. En nuestro estudio, la difusión en $\mathrm{Sr}_{3} \mathrm{Ca}_{1.18} \mathrm{Nb}_{1.82} \mathrm{O}_{9-\delta}$ requiere el transporte de tres especies catiónicas diferentes, y es responsable de la deformación plástica total, ya que el deslizamiento de junta de grano no es plausible una vez que se alcanzan condiciones de deformación en estado estacionario, por lo que no es sorprendente encontrar valores distintos de la energía de activación. Los exponentes de tensión son del orden de 2 para el $\mathrm{Sr}_{3} \mathrm{Ca}_{118} \mathrm{Nb}_{182} \mathrm{O}_{9-\delta}$ y de aproximadamente 1 para el $\mathrm{SrCe}_{0.9} \mathrm{Y}_{0.1} \mathrm{O}_{3-\delta^{\prime}}$ como se observa en la figura 8.

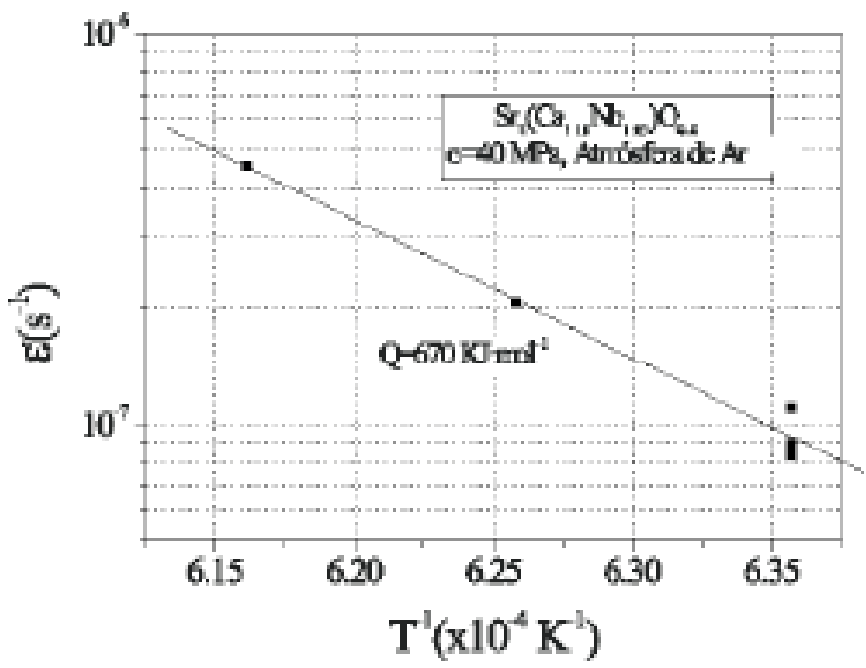

Fig. 7.- Determinación de la energía de activación del $\mathrm{SrCe}_{0.9} \mathrm{Y}_{0.1} \mathrm{O}_{3-\delta}$.

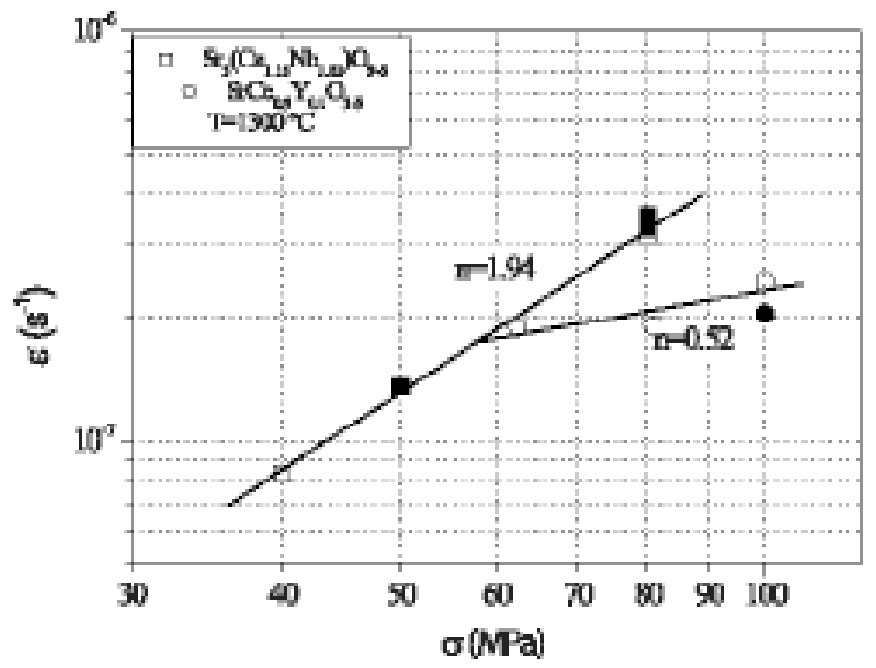

Fig. 8.- Determinación del exponente de tensiones $\mathrm{n}$ para todos los materiales estudiados. Los símbolos sólidos corresponden a ensayos en aire, y los huecos a ensayos en atmósfera de Ar.

\subsection{Caracterización de las muestras deformadas}

Tras los ensayos mecánicos se estudió el corte transversal de una muestra de $\mathrm{SrCe}_{0.9} \mathrm{Y}_{0.1} \mathrm{O}_{3-\delta}$ mediante EBSD. Debido a la fuerte textura de las muestras recibidas, es posible medir variaciones en la orientación menores que $1^{\mathrm{o}}$. En la figura 9 se representan los mapas de orientaciones obtenidos antes y después de los ensayos mecánicos. En particular, se representa el ángulo que forma en cada punto el eje $<100>$ del prototipo cúbico con la normal a la muestra (dirección de crecimiento - figura 9A). Se observa que en las muestras ensayadas se ha producido una rotación rígida de las células, como evidencia el contraste más claro e inhomogéneo de la figura 9B si la comparamos con la 9A. A las temperaturas de los ensayos realizados la viscosidad de la fase amorfa se reduce y las células pueden rotar sin deformarse, de manera que el material acomoda la deformación mediante flujo viscoso de la fase amorfa. Eventualmente las células llegarán a tocarse, produciéndose coalescencia entre ellas ya que la su orientación es casi idéntica. Cuando esto ocurre las células unidas entre sí no pueden rotar libremente, y su movimiento debe acomodarse por difusión. El mecanismo pasa entonces a ser de tipo Nabarro-Herring (11). Esto explica las variaciones en las velocidades de deformación que se observan en la figura 5 durante las 75 primeras horas de ensayo. Hasta entonces las células rotan y van coalesciendo, de manera que hay una evolución microestructural. El flujo viscoso contribuye a la deformación, y que el sistema tarda mucho tiempo en alcanzar el estado estacionario. Dryden y Wilkinson (12) proponen un modelo de flujo viscoso en el que la velocidad de deformación depende linealmente de la tensión aplicada. Refinamientos posteriores del modelo por Martínez-Fernández y col. (13) muestran que la velocidad de deformación debe disminuir más rápidamente con un mayor contenido de fase viscosa, dando lugar a determinaciones anómalas del exponente de tensión, que puede llegar a ser inferior a la unidad, como se observa en la figura 8 para el $\mathrm{SrCe}_{0.9} \mathrm{Y}_{0.1} \mathrm{O}_{3-\delta}$.
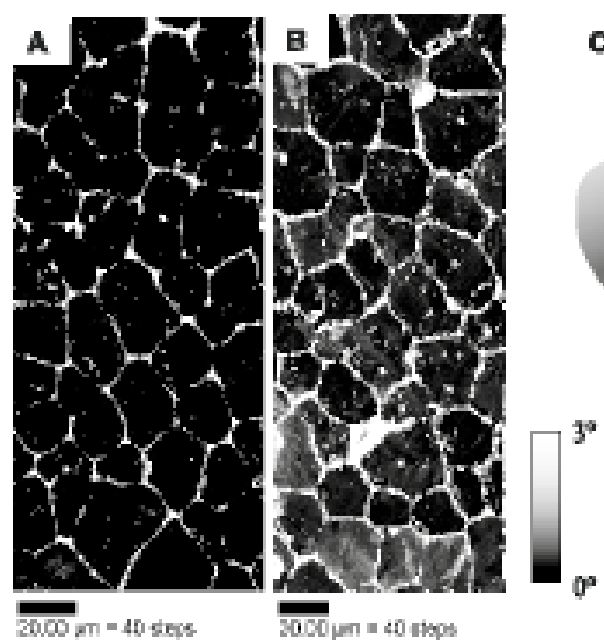

Fig. 9.- Mapa de orientaciones de una sección transversal de una muestra de $\mathrm{SrCe}_{0.9} \mathrm{Y}_{0.1} \mathrm{O}_{3-\delta}$ en la que se representa el ángulo que forma la dirección $<100>$ con la dirección de crecimiento. A) Muestra recibida, B) Muestra deformada a $90 \mathrm{MPa}$ en $\mathrm{Ar}$ a $1300^{\circ} \mathrm{C}, \mathrm{C}$ ) Esquema en el que se muestra el ángulo representado en los mapas. Se observa que los granos han rotado rígidamente tras la deformación plástica. 
Para comprobar que existe flujo en la fase viscosa, se sometió una muestra de $\mathrm{SrCe}_{0.9} \mathrm{Y}_{0.1} \mathrm{O}_{3-\delta}$ a tratamiento térmico a $1300^{\circ} \mathrm{C}$ durante $16 \mathrm{~h}$ en atmósfera de aire. En la figura 10 se observa que efectivamente la fase vítrea fluye hacia el exterior por efecto de la dilatación térmica en la muestra, distinta a la de las células; también puede observarse que se produce la coalescencia de las células.

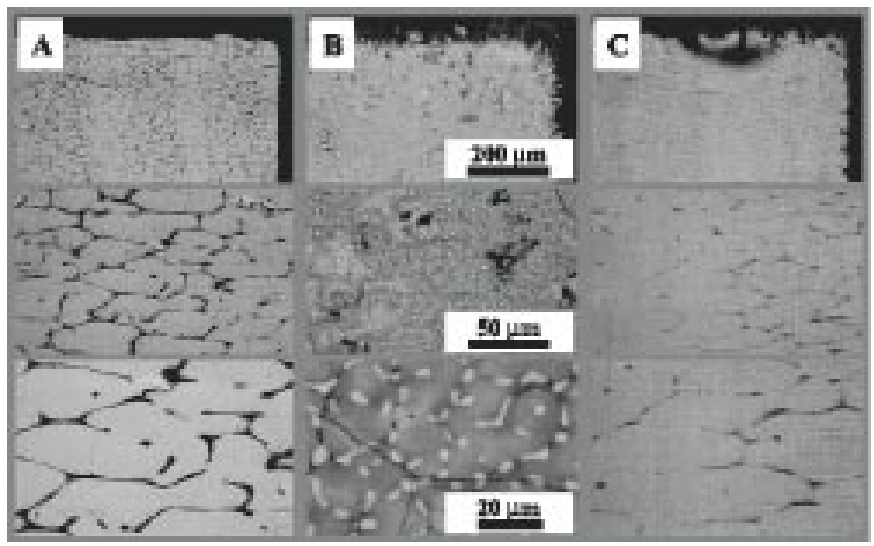

Fig. 9.- Micrografías MEB de la muestra $\mathrm{SrCe}_{0.9} \mathrm{Y}_{0.1} \mathrm{O}_{3-\delta}$ antes (A) y después del tratamiento térmico a $1300^{\circ} \mathrm{C}$ en aire. (B) superficie exterior y (C) microestructura interior. Se observa que la fase amorfa fluye hacia el exterior de la muestra.

\section{CONCLUSIONES}

Se han caracterizado microestructuralmente las perovsquitas $\mathrm{SrCe}_{0.9} \mathrm{Y}_{01} \mathrm{O}_{3-\delta}$ y $\mathrm{Sr}_{3} \mathrm{Ca}_{118} \mathrm{Nb}_{182} \mathrm{O}_{9-\delta}$ fabricadas mediante solidificación direccional. La microestructura única de estos materiales constituida por células con orientación idéntica separadas por una fase amorfa es responsable de su comportamiento plástico. Se han realizado ensayos de fluencia obteniéndose exponentes de tensión $\mathrm{n} \sim 1 \mathrm{y} \mathrm{n} \sim 2$. Para el $\mathrm{Sr}_{3} \mathrm{Ca}_{118} \mathrm{Nb}_{182} \mathrm{O}_{9-\delta}$ se ha obtenido una energía de activación de $670 \mathrm{KJ} / \mathrm{mol}$. Se ha observado que el comportamiento plástico de estos materiales es independiente de la atmósfera de trabajo. La deformación plástica produce rotación de las células, y la fase amorfa fluye hacia el exterior de la muestra. Podemos concluir que los CPAT fabricados por solidificación direccional asistida por láser deben su buen comportamiento mecánico a su microestructura única formada por células de topología compleja. La deformación plástica de estos sistemas se debe a la redistribución de la fase intercelular amorfa de menor resistencia. Como causa de dicha redistribución la fase intercelular queda expulsada en parte a la superficie del material. Para tensiones bajas dicho mecanismo de deformación no se puede acomodar por un cambio de forma de los granos y la velocidad de deformación decrece con la deformación. Para tensiones más altas, la difusión se ve favorecida de forma significativa en la dirección perpendicular a la compresión, causando coalescencia de los granos en la dirección radial, que se traduce en estados de deformación estacionarios sin dependencia de la deformación.

\section{AGRADECIMIENTOS}

Este trabajo ha sido financiado por la European Office of Aerospace Research and Development, Air Force Office of Scientific Research, Air Force Research Laboratory, bajo el Contrato No. F61775-02-WE001, y por el Ministerio de Ciencia y Tecnología, bajo el proyecto MAT2003-06085-C03-03. La caracterización microestructural mediante MEB, MET y EBSD se ha realizado en el Servicio de Microscopía Electrónica del CITIUS, Universidad de Sevilla. M.J. López Robledo y J. Ramírez Rico son becarios FPI de la Junta de Andalucía.

\section{BIBLIOGRAFÍA}

1. H. Iwahara, T. Esaka, H. Uchida y N. Maeda, "Proton Conduction in Sintered Oxides and It's Application to Steam Electrolysis for Hydrogen Production", Solid State Ionics, 3/4, 359-363, (1981).

2. H. Iwahara, en: P. Colomban (Ed.), Proton Conductors: Solids, Membranes and Gels-Materials and Devices, Cambridge University Press, Cambridge, 1992, p. 122.

3. M. J. López Robledo, A. R. Pinto-Gómez, J. Martínez-Fernández, A. R. de Arellano-López, A. Sayir, "Microestructura y Propiedades Mecánicas de Conductores Protónicos de Alta Temperatura crecidos por Fusión de Zona Flotante", Bol. Soc. Esp. Ceram. V., (2004).

4. J.P. Poirer, en "Creep of Crystals", Cambridge Earth and Science series, Cambridge (1985).

5. E.T. Park, K.C. Goretta, A.R. de Arellano-López, J.Guan, U.Balachandran, S.E. Dorris y J.L. Routbort, "High-temperature deformation of $\mathrm{BaCe}_{1-\mathrm{x}} \mathrm{Y}_{\mathrm{x}} \mathrm{O}_{3-\mathrm{x}}$ $(0.05 \leq x \leq 0.20)^{\prime \prime}$, Solid State Ionic, 117, 323-330, (1999)

6. H. Sayir. S. C. Farmer, P. Lagerlof y A. Sayir "Advances in Ceramic-Matrix Composites II", editado por L.P. Singh y N. P. Bansal, American Ceramic Soc., 53-63, (1994)

7. A Sayir and S. C. Farmer, "The effect of Microstructure on Mechanical Properties of Directionally Solidified $\mathrm{Al}_{2} \mathrm{O}_{3} / \mathrm{ZrO}_{2}\left(\mathrm{Y}_{2} \mathrm{O}_{3}\right)$ Eutectic", Acta Mater. 48, 4691- 4697, (2000)

8. H. Gervais, B. Pellesier y J. Castaing, "Machine de Fluage pour Essais en Compression a Hautes Temperatures de Materiaux Ceramiques", Rev. Int. Hautes Temp. Refract., 15, $43-47$ (1978).

9. A. J. Schwartz, M. Kumar y B. L. Adams (Eds.), “Electron Backscatter Diffraction in Materials Science", Kluwer Academic/Plenum Publishers (2000).

10. J.I.Peña y col., "Microestructure of $\mathrm{Y}_{2} \mathrm{O}_{3}$ doped $\mathrm{Al}_{2} \mathrm{O}_{3}-\mathrm{ZrO}_{2}$ eutectics grown by the laser floating zone method", J. Eur.Ceram. Soc, 22, pp. 2595-2602 (2002).

11. F.R.N. Nabarro, "Report of Conf. on Strength of Solids", Physics Society, London, p. 75. (1948)

12. J. R. Dryden, D. S. Wilkinson, "Three-Dimensional Analysis Of The Creep Due To A Viscous Grain Boundary Phase", Acta Mater. 45, 1259-1273 (1997).

13. J. Martínez-Fernández, A. Muñoz, A.R. de Arellano López, F.M. ValeraFeria, A. Domínguez-Rodríguez, M. Singh, "Microstructure-mechanical properties correlation in siliconized silicon carbide ceramics", Acta Mater 51, 3259-3275 (2003)

Recibido: 09.01.05

Aceptado: 20.06.05 